SOTIRIS KAPOTAS ${ }^{1}$ and PATRICE GUILLAUME ${ }^{2}$

'Total, Division des Techniques de l'Exploration, PO Box 47, 92069 Paris la Defense, France

${ }^{2}$ CGG

\title{
INTRODUCTION
}

Issues of accurate structural interpretation along with more accurate reservoir characterization have led the oil industry to acquire more and more 3D data over more complex geological regions. This type of strategy imposes the need of a method that will allow us to estimate velocity and depth variations for these types of environments which in turn can provide a model for seismic imaging.

We present here a new reflection tomography methodology, aimed towards macro model estimation, developed to use a discrete 3D modeler as its main structural modeling facility. A high level performance ray tracer runs over this modeler and can account for various complexities within the model. The method can deal with inversion of prestack multi 2D and post stack 3D data for macro model estimation. Velocity fields can vary linearly in the vertical or have a general lateral distribution. Results on synthetic data to date have validated the effectiveness of the method to retrieve velocity and structure information in relatively complex geological environments.

In order to deal with problems of general complexity we decided to use a 3D model builder called GOCAD. Typically, in most situations, when the geologist or the geophysicist has a limited set of data at hand, this modeler is capable of building realistic geological interfaces. Unfortunately going from smoothly defined interfaces to discontinuous (realistic) ones conventional methods for modeling the travel time arrival computations are not valid. A robust and accurate ray tracer has been developed over this modeler able to estimate travel times and their derivatives in areas of general complexity. Based on this, our inversion methodology uses extensively the geometrical topology and the functionalities of the geological modeler which allow the modification of the model directly from specific modeler parameter perturbation.

\section{STRUCTURAL MODELER}

Mallet (1989b) proposed a research product for geological model building called GOCAD. The goal of this on-going research was to develop a 3D modeling tool adapted to the specificities of earth science datasets and objects.

This modeler is based on a discrete approach to geological bodies. The whole concept starts from the recognition that our knowledge of the earth relies on a very limited set of direct data (e.g. borehole data) and on a much larger set of indirect, thus less reliable, data (e.g. seismic data). In any case, these datasets offer only a sparsely sampled information about the subsurface. This notion of sampling is at the core of the DSI (discrete smooth interpolator) developed by Mallet (1989a). This interpolator is designed to estimate a physical quantity on any network of points distributed in the Euclidian space using a triangular topology. This approach leads to a faceted model for the geology.

Concerning our needs, this modeler offers the following capabilities:

- Construction of triangulated surfaces from:

Posted points.

Digitized contour maps.

Subsurface cross-sections (seismic profiles).

- Interactive surface editing.

- Topological manipulations, such as surface intersections, removal of part of a surface, etc.

- Specification of a variety of modeling constraints across surfaces or between triangles of the same surface. Such constraints can be fault throws, layer thickness, etc. 
In brief, the model used for our inversion consists of GOCAD surfaces (faulted and/or multivalued) related to the major velocity contrasts and the macro layers in between characterized by homogeneous and continuous velocity fields of the form:

$$
\mathrm{V}_{\mathrm{v}}(\mathrm{x}, \mathrm{y}, \mathrm{z})=\mathrm{V}(\mathrm{x}, \mathrm{y})+\left(\mathrm{Z}_{\mathrm{ref}}-\mathrm{Z}\right) * \mathrm{~K}(\mathrm{x}, \mathrm{y}) \text { and } \mathrm{V}_{\mathrm{h}}(\mathrm{x}, \mathrm{y}, \mathrm{z})=(1-\alpha) \mathrm{V}_{\mathrm{v}}
$$

\section{TRAVEL TIME MODELING}

The choices made for this work in terms of geological model parametrization had a large impact on the ray-tracing technique to be applied. First, algorithms had to be developed in order to get rather fast computations of ray trajectories through several inhomogeneous, sometimes anisotropic, macro-layers. The precision on computed trajectories and traveltimes must be sufficient to solve velocity estimation problems, yet a correct balance between accuracy and computation time had to be preserved. Second, the ray-tracing algorithms should handle trajectories through structurally complex geological interfaces. Conventional bending techniques perform well on smooth, spline type, surfaces, but we have seen that such techniques are hardly adapted to GOCAD triangulated surfaces.

The choice of the bending technique, developed by Guiziou et al. (1991), involved in computing a single ray between a specified (shot, receiver) pair, has been adopted for this work. This particular ray-tracing methodology is formulated as an optimization problem applied to traveltimes. Our bending technique consists in perturbing a ray trajectory iteratively, until Fermat's stationarity criterion is met that offers a global criterion to measure the adequacy of a ray trajectory. Similarly, we have selected bending techniques over shooting techniques for the following reasons:

- Robustness: The estimation of an initial ray trajectory is based on observed data information thus preparing the bending technique to "discover" the trajectory as the computations proceed.

- Accuracy: Once an initial trajectory is available, the search for the stationary ray in the

neighbourhood of that trajectory is much more efficient by bending than by shooting.

- Performance: Seismic gather geometries are well suited to bending and continuation techniques.

Continuation enables an estimation of a good initial trajectory between the source and the

receiver from the ray. The rays corresponding to the various offsets of a CMP gather are spaced closely enough to be "deduced" one from the other, using continuation and bending.

Even though we are able to derive a quasi dynamic signature of the ray, there are situations for which bending techniques are poorer than shooting techniques. This concerns primarily ray signature problems. Indeed, the search for a stationary ray by perturbing the impact points assumes that the sequence of these points remains unchanged throughout the search. Obviously, this assumption can be violated whenever the geological model becomes structurally complicated. The only definite way to handle this problem would be to verify the adequacy of the ray trajectory upon convergence of the bending optimization. Another limitation of bending algorithms is an incorrect handling of caustics. This may reveal to be annoying when very complex models have to be raytraced. However, a correct approach to caustic problems would require expensive ray amplitude computations. Presently, the problem can be handled by the observed data, i.e. multiple travel time grids referring to a single horizon. Figures 1 and 2 show results of ray tracing over 3D triangulated models.

\section{MACRO MODEL ESTIMATION}

The main strength of seismic reflection tomography is its ability to uncover velocity field heterogeneities. Interval velocity variations which commonly occur in the range of the geological models handled by our method are two fold:

- Lateral variations, of variable amplitude and areal extension.

- Vertical variations, generally limited to a gradient.

The type of data used by our method are such to allow either for prestack Multi-2D or $3 D$ post stack (Tstack-V $\mathrm{V}_{\text {stack}}$ ) inversion both controled by coherency analysis. It should be noted that 
inversion of prestack 3D data can also be done if multi-offset travel times are available. As the tomographic problem is non-linear we need to start with a good initial model to avoid local minima and get a better velocity-depth decoupling. In our model building tool there are two ways to arrive at this initial model: by map migration or by zero-offset travel time inversion with smoothed Dix derived interval velocities.

Once a choice of raytracing has been made to drive the inversion, the second issue is the development of the model based inversion parameters. Partial derivatives with respect to model parameters must be accurately estimated in order to develop an optimization scheme. The interfaces are parameterized by control nodes governed by the inverse DSI operator allowing for three different levels of variation (XY,XYZ and $Z$ ). We have been able to compute the inverse of the DSI operator which gives the partial derivatives of each triangle vertex position with respect to each control node position. The layer velocities are represented by $B$-spline coefficients on a 2D tensor thus reducing the number of parameters for inversion. This reduction of parameters is important because of the size of the 3D real models we need to estimate.

The above mentioned inverse operator bridges the gap between the ray tracer and the travel time inversion scheme. Thus, these partial derivatives derived with the aid of ray tracing and the DSI operator, can be used in our optimization problem and lead to macro model estimation. Our optimization scheme uses the global least square Marquard-Lavenberg descent method for the solution of the system of equations. The options of local and global inversion parameters are also operational as well as the inversion of "pinch-outs" and other discontinuities.

\section{QUALITY CONTROL AND APPLICATIONS}

As with any product that uses a series of data and parameters a utility which allows for visual control of a a priori and final data must be in place. Our utilities allow for the following quality controls:

- Interactive 3D visualisation, editing and control of surface/depth parameters

- Integrated displays of velocity maps with superimposed velocity parameters.

- Integrated displays of discrepancies between modeled and measured data with superimposed zones of clearance.

- Display of CMP gathers related to velocity analysis, with superimposed measured and modeled hyperbolic and actual travel time curves.

- Evolution of coherency measure during optimization.

Several synthetic data tests have been carried out in order to validate the potential of this method. Figures 3 and 4 show depth and velocity profiles estimated by our tomographic inversion from synthetic data. We are in the process of analyzing real data sets and hope to present the results during the workshop.

\section{ACKNOWLEDGEMENTS}

The authors wish to acknowledge the commission of European Communities for financial support of the SISTRE project, involving TOTAL, CGG, TNO, and EAP, under contract No. TH $01.129 / 89$.

\section{REFERENCES}

Guiziou, J.L., Mallet, J.L, Nobili, P., 1991, 3-D Raytracing through Complex triangulated Surfaces, 61th Annual SEG Meeting expanded abstract, Houston, 1497-1500.

Mallet, J.L., 1989a, Discrete Smooth Interpolation: ACM Transactions on Graphics, vol. 8, num. 2, 121-144.

Mallet, J.L., 1989b, GOCAD: A Computer Aided Design Program for Geological Applications: Proceedings of a Nato Advanced Research workshop. A. Keith Turnur Editor, Kluwer Publishing Company. 


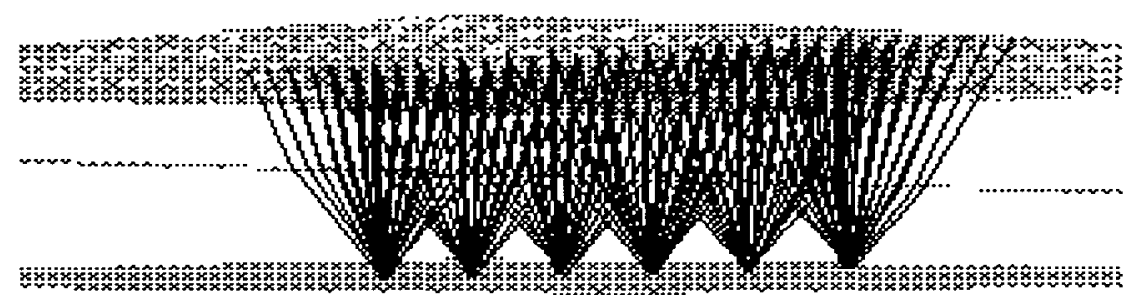

Figure $1.3 \mathrm{D}$ rays over a diping layer model

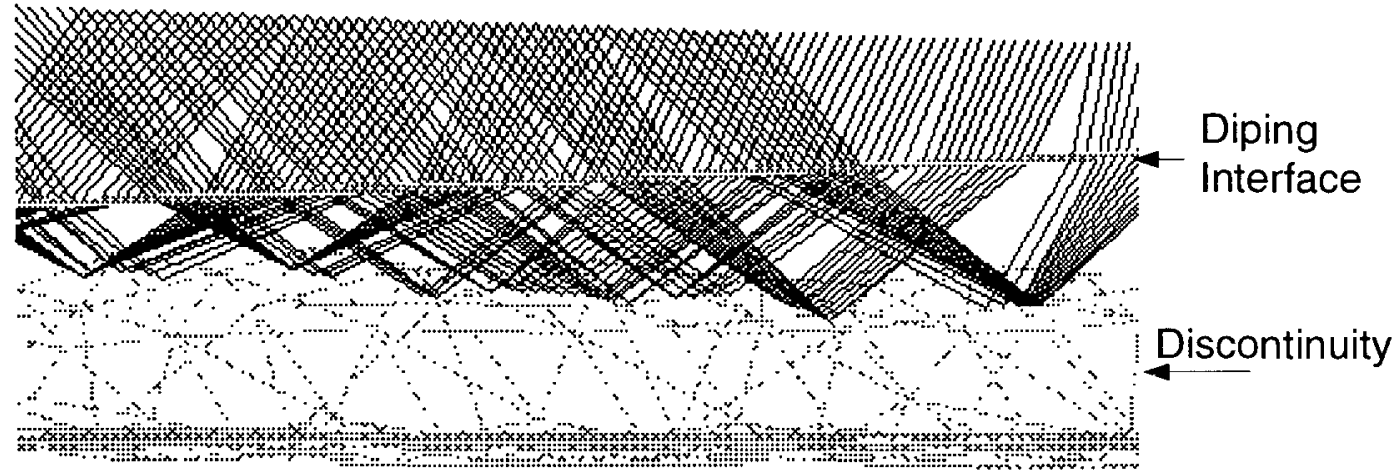

Figure 2. Rays over a complex 3D triangulated model

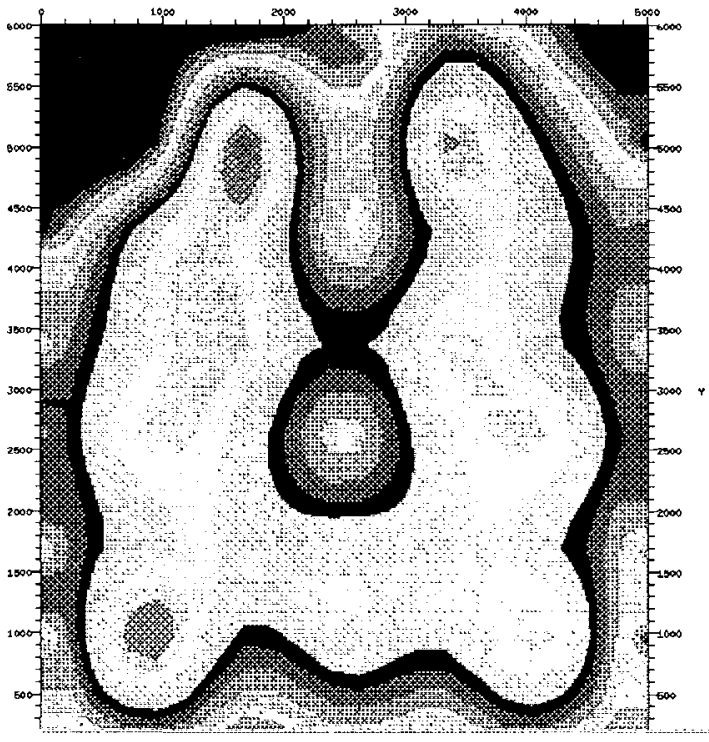

Figure 3. Interface estimated by tomographic inversion

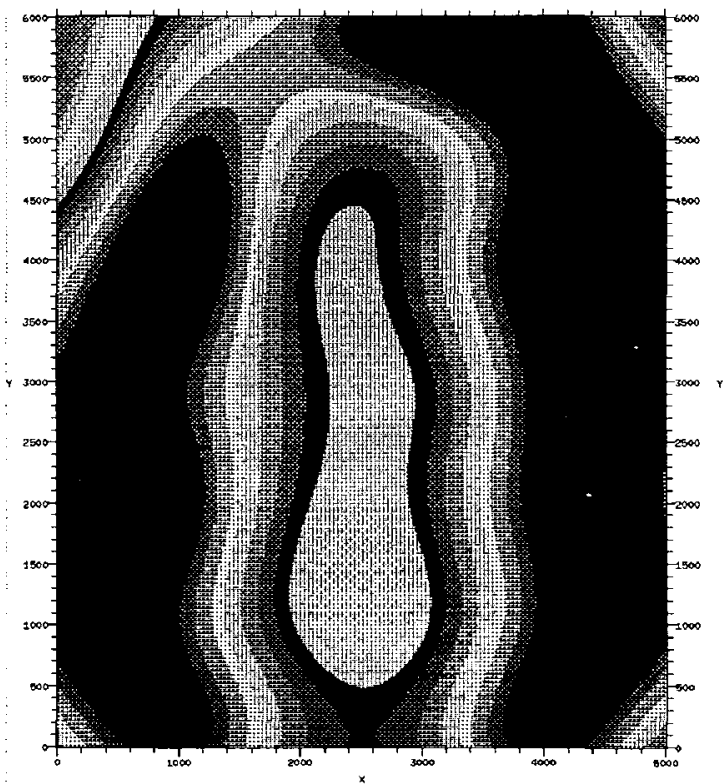

Figure 4. Interval velocity derived from 3D tomographic inversion 